\title{
Atopic Dermatitis or Hyper IgE Syndrome?
}

\author{
Ahmed $\mathbf{M}^{1}$, Kumar $\mathrm{A}^{2}$, Raj $\mathrm{D}^{3}$
}

\begin{abstract}
Eczema and skin infections are commonly encountered in children in the outpatient department. However, presence of both recurrent dermatitis and skin infections should alarm one to consider Hyper IgE syndrome (HIES) as a differential. Here we present a case of HIES in a two and a half year old female child with history of recurrent dermatitis and skin infections in the form of cold abscesses. Laboratory investigations showed markedly elevated IgE levels and high absolute eosinophils counts. Patient was treated with trimethoprim-sulfamethoxazole and supportive care. The current report highlights the fact that HIES can present in early childhood with just skin manifestations and without any significant pulmonary manifestations.
\end{abstract}

Key words: Hyper IgE syndrome, Eosinophilia

\section{Introduction}

Eczema and skin infections are commonly encountered in - children in the outpatient department. However, presence of both recurrent dermatitis and skin infections should alarm one to consider Hyper IgE syndrome (HIES) as a differential. Hyper IgE syndromes are a group of rare primary immunodeficiency disorders characterized by marked elevations in IgE levels, recurrent staphylococcal skin infections, recurrent pneumonia and chronic eczematoid dermatitis ${ }^{1}$. The skin infections are in the form of boils and furuncles which typically lack the signs of inflammation, hence referred to as "cold abscesses".

Hyper IgE syndrome is a complex immune deficiency with diverse clinical manifestations and heterogenous genetic origins ${ }^{2}$. HIES are mostly sporadic but two inherited forms are recognized, a dominant form (Type 1), caused by mutations in STAT3(Signal transducer and activator of transcription 3 ), and a recessive form (Type 2) is due to mutation in Dock-8 or cytokine sis- 8 and TYK2 ${ }^{3}$. This report highlights the fact that HIES can be suspected on the basis of the key features observed in early childhood like chronic recurrent dermatitis and skin infections.

\section{The Case}

This twoand a half year old girl was born to non-consanguineous parents with uneventful perinatal events at term gestation with a
'Dr. Minhajuddin Ahmed, Senior Resident (MD), Department of Paediatrics, RKDF Medical College Hospital \& Research Centre, Sarvepalli Radhakrishnan University, Bhopal, ${ }^{2}$ Dr. Abhay Kumar, Resident (DNB), Department of Paediatrics, Holy Family Hospital, New Delhi, ${ }^{3}$ Dr. Dinesh Raj, Consultant Paediatrician, Department of Paediatrics, Holy Family Hospital, New Delhi.

Address for correspondence:

Dr Dinesh Raj

E-mail:dr_aiims@yahoo.co.in

\section{How to cite}

Ahmed M, Kumar A, Raj D. Atopic Dermatitis or Hyper IgE Syndrome? J Nepal Paediatr Soc 2015;35(1):59-61.

doi: http://dx.doi.org/10.3126/jnps.v35i1.11383

This work is licensed under a Creative Commons Attribution 3.0 License.

\section{(c) (i)}

birth weight of $3 \mathrm{kgs}$. She was exclusively breast feed till six months of age. The child presented to the paediatric outpatient department with complaints of severe pruritis over face, hands, abdomen, ears and face since the age of 18 months and cold abscesses on buttock and thighs. The child also had frequent upper respiratory tract infections requiring hospital outpatient visits. She was admitted once with acute lower respiratory infection needing intravenous antibiotics, however the chest radiograph did not reveal any significant findings. On physical examination, the child had a dry eczematous scaly rash with crusting predominantly on cheeks, abdomen, forearm, and buttock (Fig 1 and 2 ) and cold abscess over peri-anal region 
which was fluctuant cyst like lesion, neither hot nor tender with no signs of any local or generalized inflammation (Fig 3). Vital parameters were in the normal range. Respiratory, cardiovascular, abdominal, and nervous system examination was unremarkable. On investigation, hemoglobin was $10.6 \mathrm{gm} \%$, total leukocyte count was 15700/ $\mathrm{LL}$ (polymorphs 32\%, lymphocytes $49 \%$, eosinophils $10 \%$, monocytes $8 \%$ ), platelets $9.54 \times 10^{9} / \mathrm{L}$, absolute eosinophil counts were $1531 / \mu \mathrm{L}$ and IgE level was $18,403 \mathrm{IU} / \mathrm{ml}$. Chest radiograph was normal.

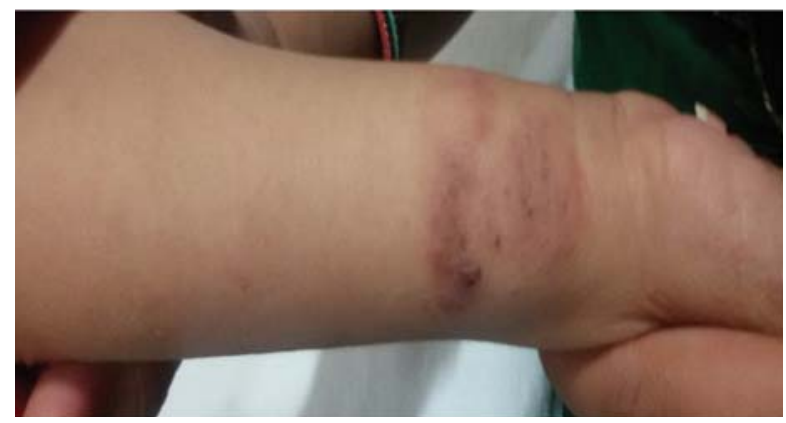

Fig 1: Dry eczematous scaly rash over wrist and lower limbs

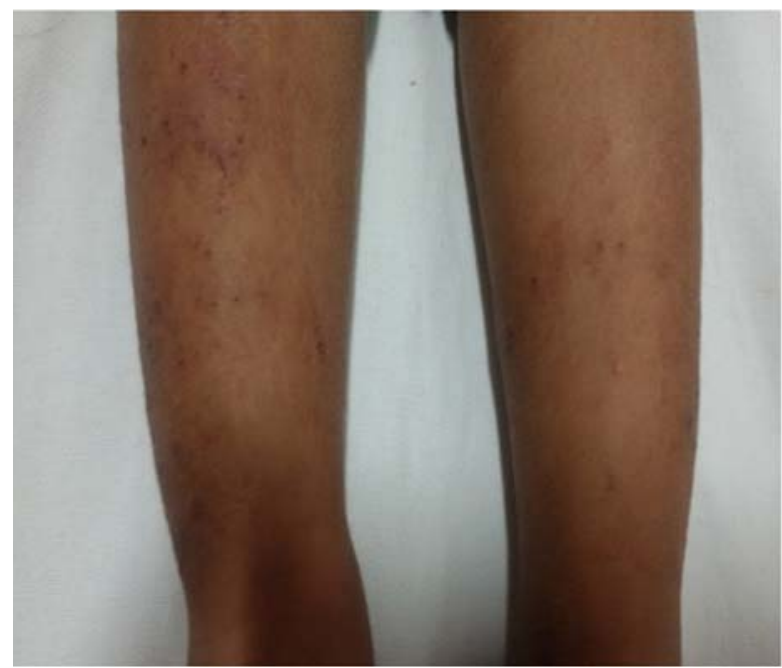

Fig 2: Dry eczematous scaly rash over lower limbs

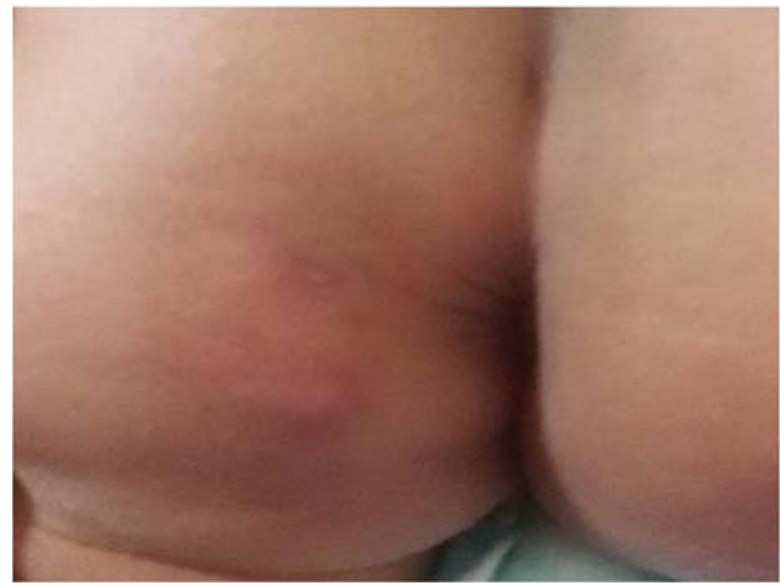

Fig 3: Cold abscess over peri-anal region

\section{Discussion}

Dermatitis and skin infections with abscesses are quiet often seen in paediatric age group leading to significant morbidity and adversel affects. Although the skin rash of HIES resembles atopic dermatitis, Hochreutener et al emphasized the importance of clinical differentiation of the HIE syndrome from atopic dermatitis because the treatment and prognosis are different ${ }^{4}$. As atopic dermatitis is the commonest cause of recurrent dermatitis in children, clinicians think of $A D$ as the likely possibility in this scenario, and often overlook the skin infections. AD is a chronic relapsing hypersensitive manifestation of skin, itching being a significant feature. An increasing trend of $A D$ is seen in India. Around $10-20 \%$ of children and $1-3 \%$ of adults are affected in developed countries with this type of skin manifestation ${ }^{5}$ In addition to atopic dermatitis, the differential diagnosis of HIE syndrome includes DiGeorge syndrome, Wiskott -Aldrich syndrome and chronic granulomatous disease. It is therefore necessary to consider these possibilities whenever a child with dermatitis and skin infections is evaluated.

To identify patients with HIES, NIH (National Institute of Health) scoring system is used, which is based on certain clinical and laboratory findings. Patients with an NIH score of 40 or more are likely to have a STAT3 mutation. Grimbacher et al prospectively evaluated patients with suspected HIES according to the NIH scoring system ${ }^{6}$. They also scored cases of atopic dermatitis and other immune deficiencies for comparison. The maximal score obtained for each age group was 25 for 0-2 years, 60 for 3-6 years, 76 for 7-12 years, and 96 for more than 18 years. The study suggested that points more than $40 \%$ of the maximal score was significantly associated with a diagnosis of HIES, points less than $20 \%$ were not observed in HIES, and diagnosis can't be ruled out for points between 20$40 \%$. In our case, the score obtained was 31 , which was well above the maximal score obtained for that age group by Grimbacher et $a^{6}$.

HIES has an incidence of 1 in 500,000 live births ${ }^{7}$ and occurs in both males and females of all ethinic groups with equal frequency ${ }^{8}$. Type 1 is a dominant form caused by heterozygous mutations in the transcription factor STAT3 involving skin, connective tissue, skeleton and characteristic facial appearance. The locus for familial autosomal dominant (AD)-HIES, STAT3 is located on chromosome $17 q 21^{5}$. Thus, a deficiency in STAT3 is a cause of sporadic and familial HIES. Type 2 HIES is an autosomal recessive (AR) syndrome ${ }^{7}$. The patients with type 2 HIES do not have skeletal and dentition abnormalities. 
In HIES, IgE levels exceeds $2000 \mathrm{IU} / \mathrm{ml}$ and may decrease with age or remain within normal range in about $20 \%$ of cases ${ }^{9}$. IgE anti staphylococcal antibodies are common and are specific. The NIH score correlates with the severity of disease and it is a useful tool to diagnose of AD- HIES rather than AR-HIES. Definitive diagnosis is established by genetic study.

As there is no definitive therapy available, so the strategy in HIES is towards prevention and management of bacterial infections. Good skin care and hygiene is necessary to prevent skin infections. Antimicrobial prophylaxis to prevent Staphylococcus aureus skin and lung infection is among the most widely used treatments options. Lifelong antibiotics and antifungals as required reported to be safe and effective. Trimethoprim-sulfamethoxazole is a safe and effective alternative to penicillin; importantly, it has anti-MRSA properties ${ }^{10}$. High-dose IV Immunoglobulin shown to lead to marked clinical improvement in patients with HIES ${ }^{11}$.

Our report highlights the fact that possibility of HIES should be considered in children presenting with recurrent eczema and skin infections. The NIH scoring system can be used in establishing the diagnosis.

\section{Conclusion}

HIES is a rare primary immunodeficiency syndrome which occurs in early childhood with triad of symptoms. This case highlights high suspicion rate to be considered for HIES in patients with recurrent chest infections with skin infections. Early diagnosis and treatment is life saving and can reduce morbidity. Trimethoprim-sulfamethoxazole is safe, effective and should be given for long term as prophylactic therapy.

\section{References}

1. Freeman AF, Holland SM. The hyper-IgE syndromes. Immunol Allergy Clin North Am2008;28:277-91.

2. E. L. Rael, R. T. Marshall and J. J. McClain, "The Hyper-IgE syndromes: lessons on nature, from bench to bedside," World Allerg Org J 2012;5(7):7987.

3. Ghaffari J, Abedain-Kenari S, Ghasemi $M$, Gohardehi F. Psoriasis in hyper IgE syndrome-a case report. Caspian J Int Med2013;4(3):735.

4. Hochreutener $\mathrm{H}$, Wuthrich B, Huwyler $\mathrm{T}$, et al. Variant of hyper- IgE syndrome: the differentiation from atopic dermatitis is important because of treatment and prognosis. Dermatologia 1991;182:7-11

5. Saito H. Much atopy about the skin: genomewide molecular analysis of atopic eczema. Int Arch Allerglmmuno/2005;137:319-25.

6. Grimbacher B, Schaffer AA, Hollad SM, Davis J, GallinJ, Malech HL, Atkinson TP, BelohradskyBH, Buckley RH, Cossu F, Espanol T, GartyBZ, Matamoros N, Myers LA, Nelson RP, Ochs HD, Renner ED, Wellonghausen N, Puck JM: Genetic linkage of hyper- IgE syndrome $t$ chromosome 4.Am J Hum Gen 1999;65:735-44.

7. De Witt CA, Bishop AB, Buescher L, Stones S. Hyperimmunoglobulin $\mathrm{E}$ : two cases and review of the literature. J Am AcadDermatol 2006;54:85565.

8. Deepa D, Kumar KA, Joshi CS, Kumar S, Pandey A. Fungal infection of gingival in a patient with hyperimmunoglobulin-E (Job's) syndrome. J Indian SocPeridontol 2012;16(2):256.

9. Y. Minegishi, M. Saito, T. Morio, et al., "Human tyrosine kinase 2 deficiency reveals its requisite roles in multiple cytokine signals involved in innate and acquired immunity," Immunity2006;25(5): 745-755.

10. Grimbacher B, Holland SM. GallinJI, Greenberg F, Hill SC, Malech HL et al. Hyper Ig E syndrome with recurrent infections- An autosomal dominant multisystem disorder. N Eng J Med 1999; 340:692702.

11. Tanaka H, Ito R, Onodera N, Waga S. Efficacy of longtermsulfamethoxaxole - trimethoprim therapy in a boy with hyperimmunoglobulin $\mathrm{E}$ syndrome. Tohoku J Exp Med 1998;186:61-66. 